\title{
Platelet microRNAs and vascular injury
}

\author{
Elena V. Dolmatova and Kathy K. Griendling \\ Division of Cardiology, Department of Medicine, Emory University, Atlanta, Ceorgia, USA.
}

\begin{abstract}
Vascular smooth muscle cell (VSMC) phenotype switching from a contractile state to a synthetic phenotype has been implicated in intimal remodeling during vascular injury. While multiple studies have focused on dedifferentiation of VSMCs, prevention of VSMC-mediated excessive repair remains poorly understood. In this issue of the $J C I$, Zeng et al. identified a mechanism by which platelet-derived microRNA-223 (miRNA-223) reverses VSMC dedifferentiation. The authors show that suppression of proliferation occurs after platelet internalization by VSMCs. Moreover, they demonstrate that miRNA-223 inhibits dedifferentiation and intimal hyperplasia in diabetic mice by decreasing PDGFR $\beta$ expression in VSMCs. Together, these results identify platelet-derived miRNA-223 as a potential therapeutic target in vascular injury.
\end{abstract}

inflammatory effect on the endothelium, in VSMCs, miRNA-22 (although not from platelets) has been shown to promote phenotypic switching and neointima formation (13). miRNA-143 and -145, in contrast, induce differentiation and repress proliferation of VSMCs $(14,15)$. Zeng et al., however, provide evidence that at least some of the miRNA-143 and -145 in VSMCs during vascular injury is likely to originate from activated platelets. These findings indicate the complexity of miRNA-mediated intercellular signaling, which is dependent on the exact miRNA, the delivery mechanism, and the recipient cell. Further investigation of the distinct roles of plateletderived miRNAs in various tissues, and even cell types within a particular tissue, is thus warranted. This knowledge can provide insight into therapeutic targets for such vascular processes as micro- and macrovascular complications of diabetes (16) and re-endothelialization and VSMC proliferation after stent placement (17).

\section{miRNA-223}

While miRNA-143 and -145 are expressed in both platelets and VSMCs, miRNA-223 expression is restricted to hematopoietic cells and is one of the most abundant miRNAs in platelets (9). It is also the only miRNA that has been linked to P2Y12 expression and reactivity to antiplatelet agents (9). Patients with diabetes were found to have lower levels of miRNA-223, resulting in higher expression of P2Y12 receptor and P-selectin, thus contributing to platelet hyperactivation (18). Moreover, lower levels of miRNA-223 were associated with a higher incidence of stroke (19) and increased mortality (20).

Although the aforementioned effects of miRNA-223 on platelet function have been studied quite extensively, knowledge of its role in other cells remains limited. Interestingly, freshly isolated endothelial cells have high levels of miRNA-223, which are lost early during culture (21). It was proposed that in vivo microRNA-223 levels in endothelial cells are maintained via microparticledependent transfer from platelets (6). Such 

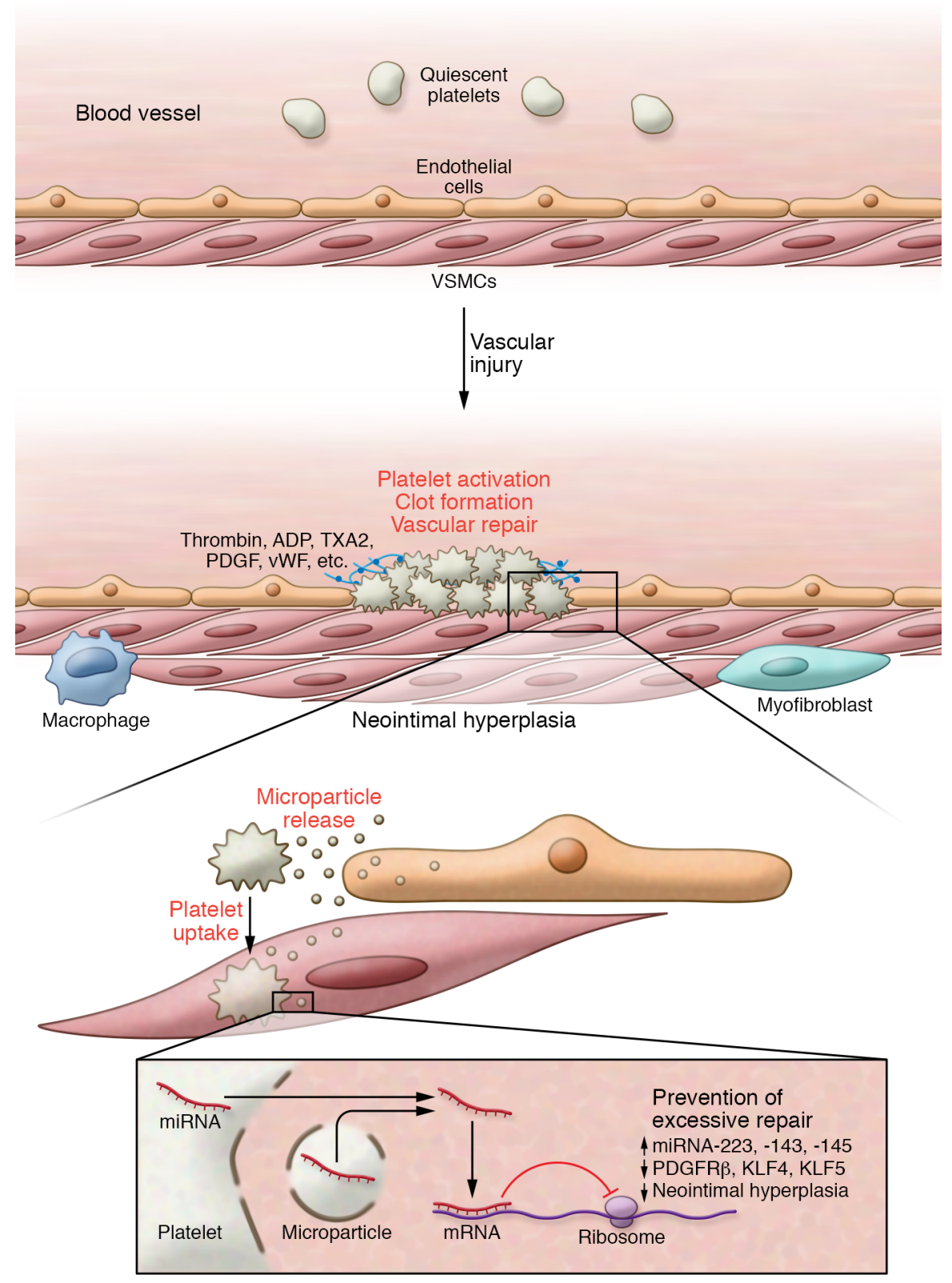

Figure 1. Summary of the possible mechanisms for platelet-derived miRNA transfer during vascular injury. With intact endothelium, platelets are quiescent. When the endothelial barrier is compromised, platelets are activated, form a clot, and release various factors promoting vessel repair They also release microparticles (MPs) containing miRNAs that are taken up by endothelial cells (and possibly other cells) and exhibit various effects on protein expression in recipient cells. Next, the platelets are taken up by VSMCs and lysed, releasing miRNAs into cytoplasm of VSMCs. In particular, miRNA-223, -143 , and -145 released in VSMC cytoplasm decrease expression of PDCFR $\beta$, KLF5, and KLF4, thereby suppressing VSMC dedifferentiation and excessive repair.

transfer had not been shown for VSMCs, however, until, in the current issue of the JCI, Zeng et al. demonstrated an important role for platelet-derived miRNA-223 in VSMC differentiation. The authors also showed that delivering the AgomiRNA-223 complex in miRNA-223-deficient mice (as a result of either genetic modifications or diabetes) can abrogate intimal hyperplasia after injury. This mal remodeling after vascular injury and should have a significant impact in the field. The use of transgenic mice with fluorescent lineage tracers allowed for an elegant demonstration of platelet uptake by VSMCs after vascular injury in vivo. Moreover, the authors identified microRNA-143, -145 , and -223 as critical in driving differentiation of VSMCs after platelet internalization. They further pinpointed KLF4, KLF5, and PDGFR $\beta$ as targets of these microRNAs. Finally, for the first time, they provided data supporting the role of miRNA-223 in intimal hyperplasia in a diabetes model, making it a compelling therapeutic target for this common condition.

Nevertheless, several important questions remain to be answered.

Zeng et al. propose a hitherto unexplored mechanism of miRNA delivery to VSMCs - by direct internalization of platelets. They provide electron micrographs of the internalized platelets and demonstrate significantly higher levels of plateletspecific miRNA-223 in VSMCs after incubation with activated platelets compared with platelet-derived microparticles. While these results are intriguing and seemingly support miRNA transfer via platelet internalization, it is also possible that both microparticle-dependent miRNA transfer and direct internalization of platelets take place upon injury. Confocal images of cocultured platelets and VSMCs from this study reveal internalized platelets along with platelets that appear to be adherent to the VSMCs. While this may represent platelets in various stages of internalization, it also allows for the possibility that some platelets supply miRNAs via microparticles. Additionally, with the described design of the in vivo experiments, one cannot distinguish between platelet uptake by VSMCs and other cell types (e.g., fibroblasts, macrophages). This is particularly important given the recent discovery that during the response to vascular injury, VSMCs can assume a macrophage-like phenotype, a transformation that may explain platelet uptake by these cells (22). More detailed studies with lineage-specific tracers or staining are needed in order to identify the proportion of platelets internalized by VSMCs. The exact cellular machinery involved in platelet internalization also remains to be described. 
Of interest, while platelet uptake by VSMCs and an increase in miRNA-223 levels coincide within hours of incubation with activated platelets, the highest levels of miRNA-143 and -145 in VSMCs were observed much later, at 24 hours. One of the possible explanations for this observation is miRNA synthesis within VSMCs themselves. Further work is needed, however, to elucidate the exact mechanism of this delayed signaling potentially responsible for continuous prevention of neointima formation.

Finally, the authors demonstrate an effect of high glucose on the levels of selected miRNAs in platelets. It is important to know, however, whether other microRNAs as well as mRNAs are affected. Currently, the data on this subject remain controversial (23) and will require further investigation. Unraveling the mechanism by which glucose exhibits this effect may also reveal additional therapeutic targets for prevention of vascular complications in diabetes.

Zeng et al. complete their study by proposing a potential role for the described pathways in other vascular pathologies, such as atherosclerosis. While this concept is intriguing, further work is needed to confirm this hypothesis. Still, the findings presented in this article have uncovered a potentially targetable pathway in a field of utmost public health significance.

\section{Acknowledgments}

KKG is supported by NIH grant HLP01095070, and EVD is supported by NIH grant 5T32HL007745-24.

Address correspondence to: Kathy Griendling, Emory University, Division of Cardi- ology, Department of Medicine, 101 Woodruff Circle, 308A WMB, Atlanta, Georgia 30322, USA. Phone: 404.727.3364; Email: kgriend@emory.edu.

1. Broos K, Feys HB, De Meyer SF, Vanhoorelbeke $\mathrm{K}$, Deckmyn H. Platelets at work in primary hemostasis. Blood Rev. 2011;25(4):155-167.

2. Provost P. The clinical significance of platelet microparticle-associated microRNAs. Clin Chem Lab Med. 2017;55(5):657-666.

3. Landry P, Plante I, Ouellet DL, Perron MP, Rousseau G, Provost P. Existence of a microRNA pathway in anucleate platelets. Nat Struct Mol Biol. 2009;16(9):961-966.

4. Provost P, Dishart D, Doucet J, Frendewey D, Samuelsson B, Rådmark O. Ribonuclease activity and RNA binding of recombinant human Dicer. EMBO J. 2002;21(21):5864-5874.

5. Gatignol A, Buckler-White A, Berkhout B, Jeang KT. Characterization of a human TAR RNA-binding protein that activates the HIV-1 LTR. Science. 1991;251(5001):1597-1600.

6. Laffont B, et al. Activated platelets can deliver mRNA regulatory Ago $2 \cdot$ microRNA complexes to endothelial cells via microparticles. Blood. 2013;122(2):253-261.

7. Zeng Z, et al. Platelet-derived miR-223 promotes a phenotypic switch in arterial injury repair. J Clin Invest. 2019;129(3):1372-1386.

8. Plé H, Landry P, Benham A, Coarfa C, Gunaratne PH, Provost P. The repertoire and features of human platelet microRNAs. PLOS ONE. 2012;7(12):e50746.

9. Pordzik J, et al. The potential role of plateletrelated microRNAs in the development of cardiovascular events in high-risk populations, including diabetic patients: a review. Front Endocrinol (Lausanne). 2018;9:74.

10. Jansen F, et al. MicroRNA expression in circulating microvesicles predicts cardiovascular events in patients with coronary artery disease. J Am Heart Assoc. 2014;3(6):e001249.

11. Harris TA, Yamakuchi M, Ferlito M, Mendell JT, Lowenstein CJ. MicroRNA-126 regulates endothelial expression of vascular cell adhesion molecule 1. Proc Natl Acad Sci U S A. 2008;105(5):1516-1521.
12. Gidlöf $\mathrm{O}$, et al. Platelets activated during myocardial infarction release functional miRNA, which can be taken up by endothelial cells and regulate ICAM1 expression. Blood. 2013;121(19):3908-3917, S1.

13. Yang F, et al. miR-22 Is a novel mediator of vascular smooth muscle cell phenotypic modulation and neointima formation. Circulation. 2018;137(17):1824-1841.

14. Cheng Y, et al. MicroRNA-145, a novel smooth muscle cell phenotypic marker and modulator, controls vascular neointimal lesion formation. Circ Res. 2009;105(2):158-166.

15. Cordes KR, et al. miR-145 and miR-143 regulate smooth muscle cell fate and plasticity. Nature. 2009;460(7256):705-710.

16. La Marca V, Fierabracci A. Insights into the diagnostic potential of extracellular vesicles and their miRNA signature from liquid biopsy as early biomarkers of diabetic micro/macrovascular complications. Int J Mol Sci. 2017;18(9):E1974.

17. Feinberg MW. Healing the injured vessel wall using microRNA-facilitated gene delivery. J Clin Invest. 2014;124(9):3694-3697.

18. Fejes Z, et al. Hyperglycaemia suppresses microRNA expression in platelets to increase P2RY12 and SELP levels in type 2 diabetes mellitus. Thromb Haemost. 2017;117(3):529-542.

19. Duan X, et al. Detection of platelet microRNA expression in patients with diabetes mellitus with or without ischemic stroke. J Diabetes Complications. 2014;28(5):705-710.

20. Keller T, et al. Improved risk stratification in prevention by use of a panel of selected circulating microRNAs. Sci Rep. 2017;7(1):4511.

21. Shi L, et al. MicroRNA-223 antagonizes angiogenesis by targeting $\beta 1$ integrin and preventing growth factor signaling in endothelial cells. Circ Res. 2013;113(12):1320-1330.

22. Shankman LS, et al. KLF4-dependent phenotypic modulation of smooth muscle cells has a key role in atherosclerotic plaque pathogenesis. Nat Med. 2015;21(6):628-637.

23. Nührenberg TG, et al. Uncontrolled diabetes mellitus has no major influence on the platelet transcriptome. Biomed Res Int 2018;2018:8989252. 